\title{
Nonvolatile voltage-controlled magnetization reversal in a spin-valve multiferroic heterostructure
}

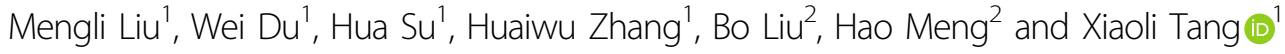

\begin{abstract}
Pure voltage-controlled magnetism, rather than a spin current or magnetic field, is the goal for next-generation ultralow power consumption spintronic devices. To advance toward this goal, we report a voltage-controlled nonvolatile $90^{\circ}$ magnetization rotation and voltage-assisted $180^{\circ}$ magnetization reversal in a spin-valve multiferroic heterostructure. Here, a spin valve with a synthetic antiferromagnetic structure was grown on a (110)cut $\mathrm{Pb}\left(\mathrm{Mg}_{1 / 3} \mathrm{Nb}_{2 / 3}\right)_{0.7} \mathrm{Ti}_{0.3} \mathrm{O}_{3}$ (PMN-PT) substrate, in which only the magnetic moment of the free layer can be manipulated by an electric field (E-field) via the strain-mediated magnetoelectric coupling effect. As a result of selecting a specified PMN-PT substrate with defect dipoles, nonvolatile and stable magnetization switching was achieved by using voltage impulses. Accordingly, a giant, reversible and nonvolatile magnetoresistance modulation was achieved without the assistance of a magnetic field. In addition, by adopting a small voltage impulse, the critical magnetic field required for complete $180^{\circ}$ magnetization reversal of the free layer can be tremendously reduced. A magnetoresistance ratio as large as that obtained by a magnetic field or spin current under normal conditions is achieved. These results indicate that E-field-assisted energy-efficient in-plane magnetization switching is a feasible strategy. This work is significant to the development of ultralow-power magnetoresistive memory and spintronic devices.
\end{abstract}

\section{Introduction}

Magnetoresistive random access memory (MRAM) is regarded as the most promising advance in nextgeneration information storage technologies ${ }^{1-3}$. It has attractive advantages such as fast operation, high density, nonvolatility, infinite endurance, and low power consumption $^{4,5}$. In typical MRAM devices, the information writing process is carried out with a magnetic field produced by a current ${ }^{6,7}$. Considerable energy consumption overheating are drawbacks encountered during the magnetization switching process. This has hindered the further development of MRAM devices. Thus, increasing attention has been given to spin-transfer torque (STT)

\footnotetext{
Correspondence: Xiaoli Tang (tangtang1227@uestc.edu.cn)

${ }^{1}$ State Key Laboratory of Electronic Thin Films and Integrated Devices, University of Electronic Science and Technology of China, Chengdu, Sichuan 610054, China

${ }^{2}$ Key Laboratory of Spintronics Materials, Devices and Systems of Zhejiang Province, Hangzhou 311305, China
}

and spin-orbit torque (SOT) due to their reduced power consumption and high storage density, in which a spinpolarized current is utilized to control magnetization switching ${ }^{8-11}$. However, decreasing the threshold current density by approximately one order of magnitude in present-day MRAMs is consistently being pursued to meet the requirements of integrated semiconductor techniques. Recently, the integration of spintronics and multiferroics has been studied as a leading candidate for developing low-power spintronic devices in numerous studies $^{12-16}$. Using the strain-mediated converse magnetoelectric (CME) coupling effect, the magnetization direction of the ferromagnetic (FM) layer in FM/ferroelectric (FE) heterostructures can be controlled by a pure electric field (E-field) instead of a magnetic field or a large spin current. This approach offers a dramatic way to construct energy-efficient MRAM devices.

A giant magnetoresistance (GMR) spin valve (SV) or magnetic tunnel junction (MTJ) normally acts as a 
memory cell in magnetic storage ${ }^{17-19}$. They have two FM layers separated by a thin nonmagnetic (NM) layer or a thin tunnel layer ${ }^{20-22}$. The magnetoresistance (MR) depends on the relative magnetization orientation between the two FM layers. The magnetic moment of a pinned layer is pinned by an antiferromagnetic (AFM) layer. The free layer can be reversed by a magnetic field, resulting in parallel and antiparallel configurations between the two FM layers. These correspond to the low and high MR states, respectively. It has been demonstrated recently that the magnetization of the free layer in SV or MTJ multiferroic heterostructures can be regulated by using an E-field via the strain-mediated ME effect, which offers attractive potential for the development of ultralow-power consumption spintronic devices ${ }^{23-26}$. In particular, a large, reversible and nonvolatile E-fieldmodulated MR of approximately $55 \%$, which is as large as a quarter of the tunnel magnetoresistance (TMR) ratio, is realized without the assistance of a magnetic field in an $\mathrm{MTJ} / \mathrm{FE}$ heterostructure ${ }^{23}$. However, according to previous reports ${ }^{23-27}$, an approximate half magnitude of the MR ratio can be modulated by an E-field since the strain only induces a $90^{\circ}$ magnetization rotation for the free layer. Although several schemes have been theoretically and experimentally proposed to achieve $180^{\circ}$ magnetization switching, special structures ${ }^{28}$ and two strain fields ${ }^{29}$ were adopted to overcome the switching barrier in the $90^{\circ}$ direction. They cannot completely match the structure applied in MRAM. Furthermore, the tunability of such an MR is limited and volatile in SV/FE heterostructures ${ }^{24-26}$, which can only be achieved with a continued E-field. These are the major challenges for the development of low-power MRAM devices.

In the present work, we report intermediate and complete magnetization reversal by an E-field with nonvolatile manipulation in an SV memory cell. An FM material $\mathrm{Co}_{84} \mathrm{Fe}_{16}$ with a positive saturation magnetostriction coefficient $(\lambda)$ was selected as the free layer in the SV to achieve strong ME coupling. A specific and a normal PMN-PT with and without defect dipoles, respectively, were selected as the substrates. The specific PMN-PT was adopted to produce a nonvolatile strain, as reported in our previous work ${ }^{30}$. Through ME coupling, a nonvolatile $90^{\circ}$ magnetization rotation and a complete $180^{\circ}$ magnetization rotation were achieved. For the $90^{\circ}$ rotation, only a half magnitude of the GMR ratio was achieved. This is unfavorable for storage. A $180^{\circ}$ magnetization reversal of the free layer is the ultimate pursuit in designing memory devices to achieve the largest MR. Complete magnetization switching with the largest MR was achieved by adopting a small voltage impulse to reduce the switching magnetic field. This breaks the significant obstacle for decreasing the magnetic field or the spin current in the writing procedure of magnetic memory and opens up a new avenue for developing E-field-controlled low powerconsuming MRAM.

\section{Materials and methods}

In this experiment, an optimized spin-valve $\mathrm{Ta}(5) / \mathrm{CoFe}$ (12)/Cu (3)/CoFe (3)/Ru (0.8)/CoFe (3)/IrMn (15)/Ta (5) $(\mathrm{nm})$ with a synthetic antiferromagnetic (SAF) structure was selected as the memory cell. It was deposited on (110)-cut $\mathrm{FE}$ single crystal $\mathrm{Pb}\left(\mathrm{Mg}_{1 / 3} \mathrm{Nb}_{2 / 3}\right)_{0.7} \mathrm{Ti}_{0.3} \mathrm{O}_{3}$ (PMN-PT) substrates by employing magnetic sputtering at room temperature. Throughout the deposition, the base vacuum was pumped to $5 \times 10^{-8}$ Torr, and the sputtering powers for the SV were $50 \mathrm{~W}$, with an Ar pressure of 3 mTorr. A constant magnetic field of 300 Oe was applied along the [001] direction of the PMN-PT. This induced an easy axis of the free layer and pinned the direction of the pinning layer along the [001] direction. Magnetization was tested by employing a vibrating sample magnetometer. The in-plane strain-E characteristics of the PMN-PT substrate were tested by binding a resistance strain gauge to the PMN-PT substrate, and the detection was conducted by using a strain gauge (WSMC1-1, Sigmar Corporation). The GMR measurements were performed using a standard four-probe configuration. All the tests were performed at room temperature.

\section{Results and discussion}

The hybrid SV/FE heterostructure structure is schematically shown in Fig. 1a. The GMR ratio is approximately $5.5 \%$, and the exchange bias field is above 1000 Oe. The SAF structure of $\mathrm{CoFe} / \mathrm{Ru} / \mathrm{CoFe}$ is chosen to produce a large exchange bias field ${ }^{31}$, while its magnetization should be fixed without being affected by the CME from the PMN-PT substrate. Figure 1b displays the magnetic hysteresis $(\mathrm{M}-\mathrm{H})$ loops of the SV heterostructure with and without an E-field. In this part, a normal PMN-PT substrate without a nonvolatile strain was chosen. The strain shows a linear increase with the voltage and decreases to zero when the voltage is released. The measured magnetic field is applied along the [001] direction. The magnetization directions of the CoFe layers are illustrated by the arrows shown in the inset of Fig. 1b. Two black arrows symbolize the magnetization directions of CoFe layers in SAF, while the blue and red arrows are employed to distinguish magnetization states of the bottom CoFe free layer without and with applying the E-field. When the magnetic field (values above $3000 \mathrm{Oe}$ ) is increased to saturate the SAF layers, the magnetization directions of all the CoFe layers become parallel to each other. The value of magnetization $\mathrm{M}$ includes contributions from all of the CoFe layers that are close to the maximum. As the magnetic field decreases, the two CoFe layers with the same thickness in the SAF are antiparallel to each other, 
(a)
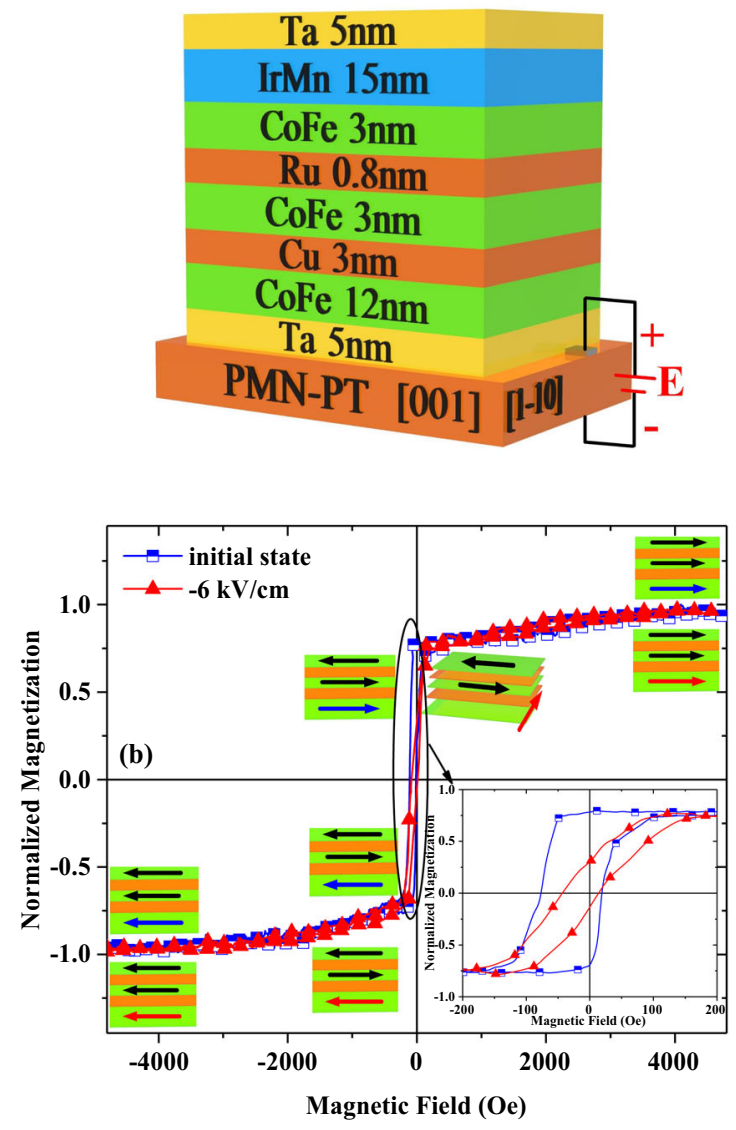

Fig. $1 \mathrm{M}-\mathrm{H}$ loops of the spin-valve multiferroic heterostructure. a Schematic of the spin-valve multiferroic heterostructure. $\mathbf{b} \mathrm{M}-\mathrm{H}$ loops of the heterostructure measured at the initial state and $-6 \mathrm{kV} /$ $\mathrm{cm}$ with the applied magnetic field along the [001] direction. The arrows symbolize the magnetization directions. The inset shows the minor loops for the switching.

which eliminates their $M$. Then, the $M$ in the low-field region depends only on the magnetization direction of the free layer. According to Fig. 1b, the sample, measured without applying the E-field, displays a square loop. Conversely, upon applying an E-field of $-6 \mathrm{kV} / \mathrm{cm}$, the loop becomes slanted, and the normalized remnant magnetization decreases significantly to nearly zero. This result confirms that the magnetic moment of the free layer rotates toward the [1-10] direction via CME coupling ${ }^{13-16,30,32,33}$. This indicates that an orthogonal configuration between the free and pinned layers can be accomplished by the E-field. Nonetheless, when the E-field is released, this orthogonal configuration cannot be retained. It reverts back to its initial parallel configuration. The stored information is lost. Hence, the method of achieving the nonvolatile E-field manipulation of GMR in a multiferroic heterostructure is highly indispensable in memory devices.

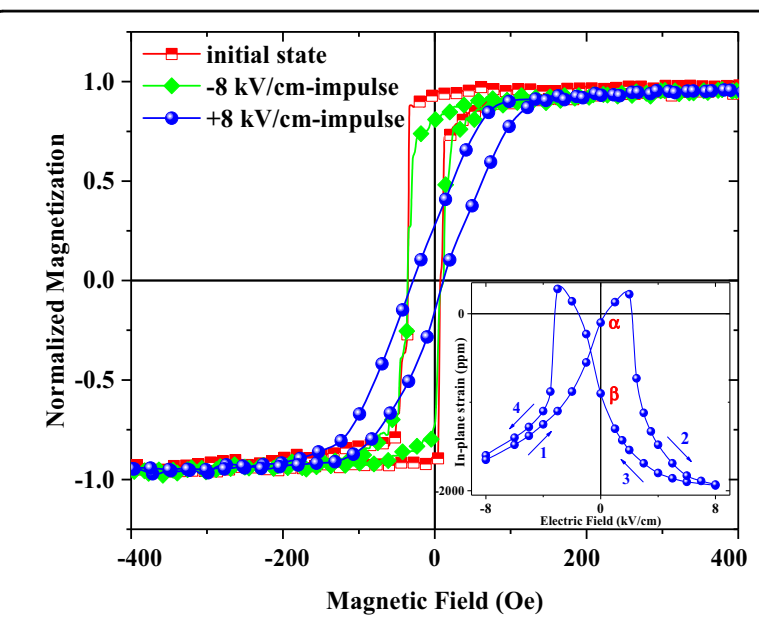

Fig. $2 \mathrm{M}-\mathrm{H}$ loops of the free layer in the spin valve. $\mathrm{M}-\mathrm{H}$ loops measured at the initial state and voltage impulses of $\pm 8 \mathrm{kV} / \mathrm{cm}$. The inset shows the in-plane strain-E loop along the [001] direction.

To achieve nonvolatile magnetization modulation, a specified FE single-crystal PMN-PT with defect dipoles was adopted. Its in-plane strain-E curve along the [001] direction was implemented by cycling the E-field between $\pm 8 \mathrm{kV} / \mathrm{cm}$. As shown in the inset of Fig. 2, the curve displays an asymmetric butterfly-like loop. In contrast to the typically symmetric butterfly-like strain curve ${ }^{32,34}$, the defect dipoles in this specific PMN-PT could induce a shift in the S-E curve. In addition, the residual strains generated by defect dipoles are more stable than those relying on the FE domain's polarization switching path ${ }^{35-38}$. Detailed analyses have been reported ${ }^{30}$. Notably, when the E-field of $-8 \mathrm{kV} / \mathrm{cm}$ is removed, the strain decreases to near zero (state $\alpha$ ), leaving almost no residual strain. Nonetheless, after applying a voltage-impulse of $+8 \mathrm{kV} / \mathrm{cm}$, a large residual strain of $-600 \mathrm{ppm}$ is achieved (state $\beta$ ). This provides an effective uniaxial magnetic anisotropy field $H_{e f f}$ expressed as follows ${ }^{33}$ : $H_{e f f}=\frac{3 \lambda\left(\sigma_{001}-\sigma_{1-10}\right)}{M_{S}}$, where $\sigma_{001}$ and $\sigma_{1-10}$ correspond to the in-plane piezo-stress values along the [001] and [1-10] directions, $\lambda$ is the magnetostriction constant, and $M_{S}$ refers to the saturation magnetization. Since the $\lambda$ of $\mathrm{CoFe}$ is positive and $\sigma_{001}-\sigma_{1-10}$ is negative, a $H_{e f f}$ that respects an effective field along the [1-10] direction is introduced. This can effectively turn the magnetic moment of the free layer toward the [1-10] direction via the strain-mediated ME effect. In the following experiment, a residual compressive strain was applied to obtain an E-controlled GMR.

As shown in Fig. 2, a typical square shape of the $\mathrm{M}-\mathrm{H}$ curve is obtained at the initial state. This is attributable to the easy magnetization axis of the free layer, which is produced along the [001] direction. When a voltage impulse of $+8 \mathrm{kV} / \mathrm{cm}$ is applied, a residual compressive strain is achieved. Then, a slanted and slim loop is observed. This indicates that a hard axis is induced along 


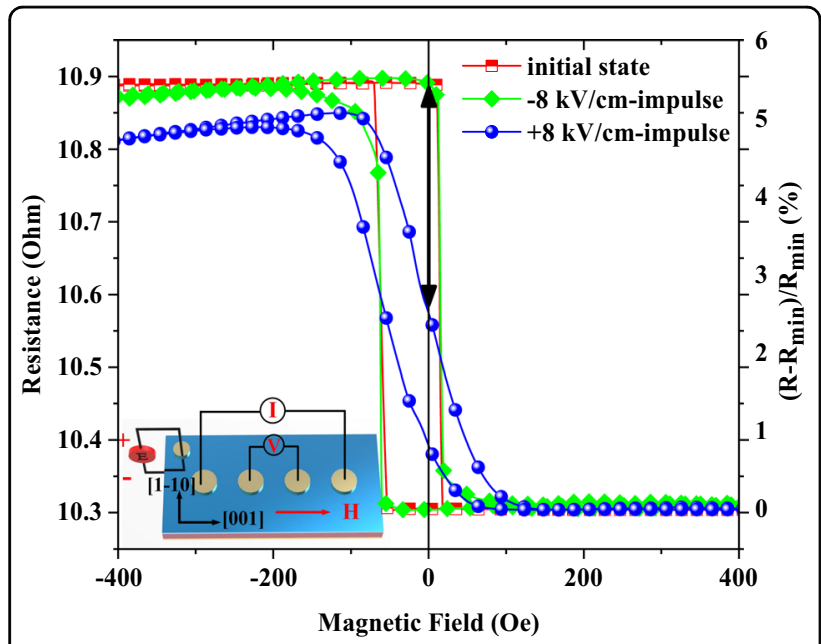

Fig. 3 The GMR curves of the spin valve. The GMR curves measured at the initial state and voltage impulses of $\pm 8 \mathrm{kV} / \mathrm{cm}$. The inset shows the schematic of the test.

the [001] direction through the residual strain. In contrast, when a pulse of $-8 \mathrm{kV} / \mathrm{cm}$ is applied, the residual compressive strain is eliminated. The magnetization direction of the free layer returns to its initial magnetic state. Therefore, the orthogonal and parallel magnetic configurations, corresponding to the relatively high and low magnetoresistances, can be easily modulated by a suitable voltage-pulse process. Additionally, these states can be retained without applying the E-field. This is a significant precondition for low-power storage.

Moreover, the E-field-controlled GMR in the SV heterostructure was investigated, as shown in Fig. 3. The testing current and magnetic field were applied along the [001] direction, as illustrated in the inset of Fig. 3. At the initial state, we first achieved its MR effect under normal measuring conditions in which a magnetic field was applied from +400 Oe to -400 Oe. The magnetic moment of the free layer is switched from a parallel to an antiparallel configuration relative to the pinned layer. The minimum value of $M R$ is achieved in the parallel configuration, and the maximum MR is achieved in the antiparallel configuration. Then, an MR effect of $5.5 \%$ is obtained by using the formula $\left(R_{\text {Max }}-R_{\text {Min }}\right) / R_{\text {Min }} \times 100 \%$. Subsequently, the MR curves were measured under $\pm 8 \mathrm{kV} / \mathrm{cm}$ voltage impulses. After the $+8 \mathrm{kV} / \mathrm{cm}$ pulse is applied, a negative residual strain along [1-10] is produced (according to the $\beta$ point in the inset of Fig. 2). It modulates the magnetization direction of the free layer rotating from the [001] to [1-10] direction, yielding half the magnitude of the MR ratio at zero magnetic field. This is due to the nearly $90^{\circ}$ magnetization orientation between the free and pinned layers without any magnetic field. If the magnetic moment needs to revert to the initial state, only a $-8 \mathrm{kV} / \mathrm{cm}$ pulse is required to release the residual strain, according to the $\alpha$ point in the inset of

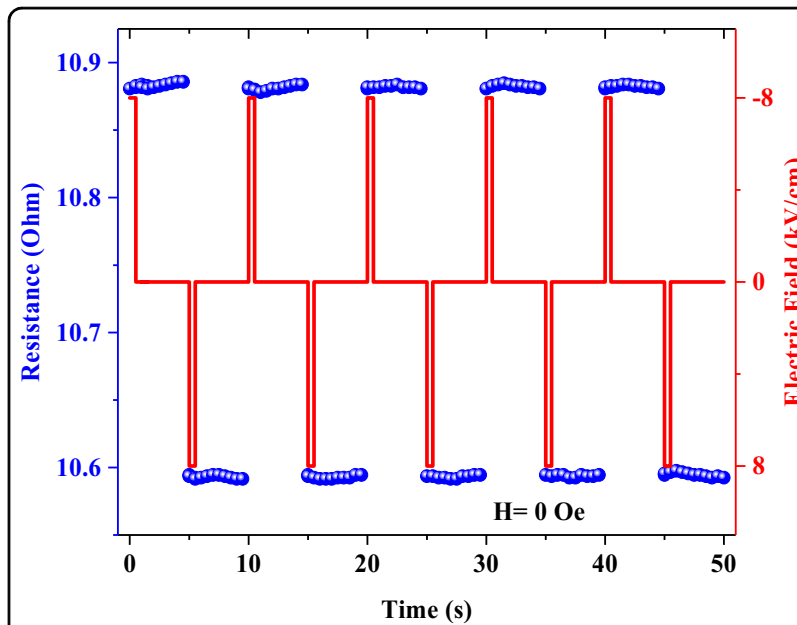

Fig. 4 Reversible and repeatable MR modulation. The high/low MR states at zero magnetic field observed under voltage impulses of $\pm 8 \mathrm{kV} / \mathrm{cm}$.

Fig. 2. Accordingly, the magnetic configuration is reversibly switched between antiparallel and orthogonal at zero magnetic field. For this condition, two stable MR values are observed, as illustrated by the black double-headed arrow in Fig. 3. The voltage-impulse-modulated MR effect is $2.5 \%$ when using $\left(R_{\mathrm{Max}}-R_{\mathrm{Min}}\right) / R_{\mathrm{Min}} \times 100 \%(\mathrm{H}=0 \mathrm{Oe})$. Furthermore, a dynamic, large and nonvolatile voltageimpulse-induced MR modulation is achieved without the assistance of a magnetic field and is displayed in Fig. 4. The pulse duration is $10 \mu \mathrm{s}$. By switching the voltage impulse between $\pm 8 \mathrm{kV} / \mathrm{cm}$, two distinct, stable, and repeatable MR states, which correspond to a giant MR effect of $2.5 \%$, are achieved. This large and nonvolatile E-field-controlled MR manipulation in the SV is noticeably more distinguished than the small and volatile modulations observed in previous reports ${ }^{24-26}$ regarding SV systems. This is a basic prerequisite to produce attractive low-power-consumption spintronic storage devices.

Even though the large and nonvolatile E-fieldcontrolled MR manipulation in our present experiments is noticeably more distinguished than in previous reports $^{24-26}$, a complete $180^{\circ}$ magnetization reversal of the free layer to achieve the largest MR is a consistent pursuit in research regarding memory devices. As mentioned above $\mathrm{e}^{29}$, the localized strain generated by one pair of electrodes on the PMN-PT substrate can only rotate the magnetization direction of the free layer within $90^{\circ}$. To overcome the barrier in the $90^{\circ}$ direction, a low E-field-assisted magnetization reversal based on a normal structure is proposed herein to meet the demands of low power and large MR simultaneously. Figure 5a shows the MR curves of the free layer measured at the initial and voltage-impulse-assisted states. The change in the applied magnetic field from +100 Oe to -200 Oe yields a 

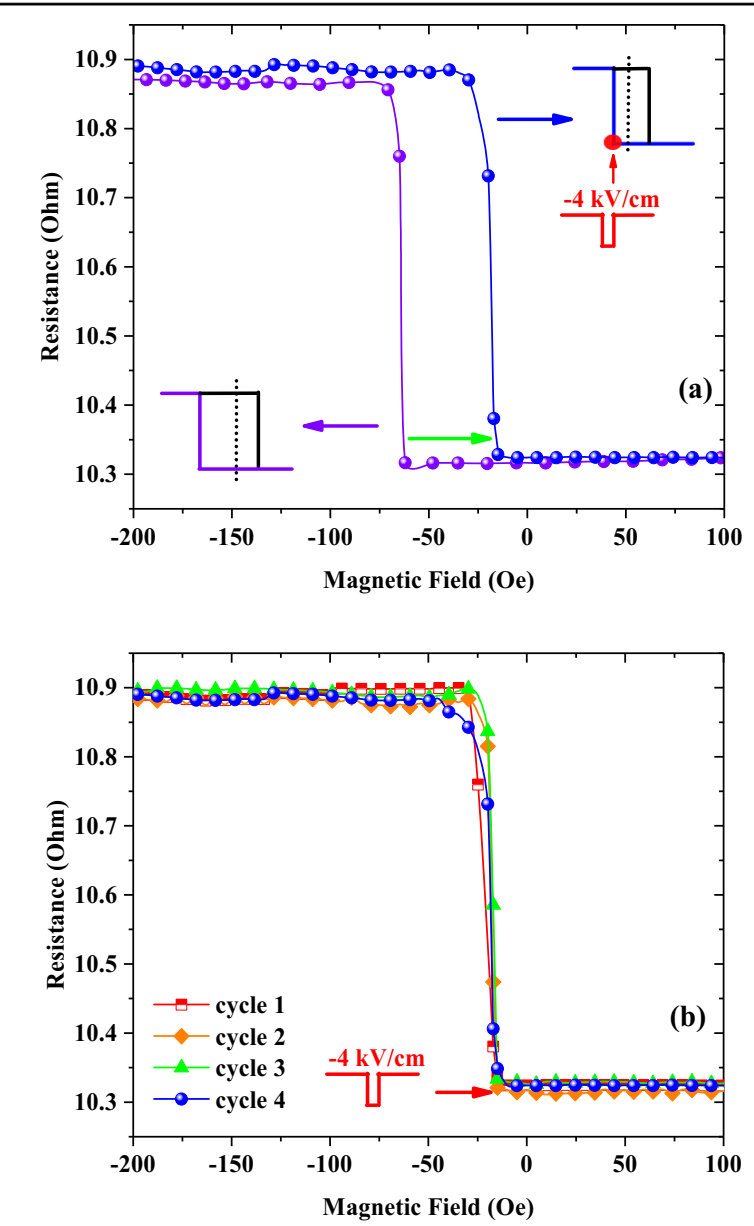

Fig. 5 Voltage-impulse-assisted $180^{\circ}$ magnetization reversal. a The MR curves of the free layer measured at the initial and voltageimpulse-assisted states. The applied magnetic field is decreased from positive to negative fields. The inset shows the whole schematics of MR curves achieved at the initial state (purple curve) and with a $10 \mu \mathrm{s}$ pulse applied at -15 Oe (blue curve). The dotted line represents the position of the zero field. $\mathbf{b}$ Repeatable MR curves of the free layer measured at voltage-impulse-assisted states.

switch from low to high MR states. In the initial state (purple data), a large magnetic field of -65 Oe is required for magnetization reversal from a parallel to an antiparallel configuration. This is greater than the magnetic field required for a reversal from an antiparallel to a parallel configuration because of the coupling field between the pinned and free layers. This issue occurs not only in GMR but also in STT and SOT spintronic devices. The applied magnetic field or spin-polarized current must be increased to ensure switching between the two states of storage. This phenomenon hinders the development of low-power-consuming MRAM devices. Fortunately, a small voltage impulse can play a vital role if it is introduced in this reversal process under proper conditions. In the measurement process, a $-4 \mathrm{kV} / \mathrm{cm}$ pulse is
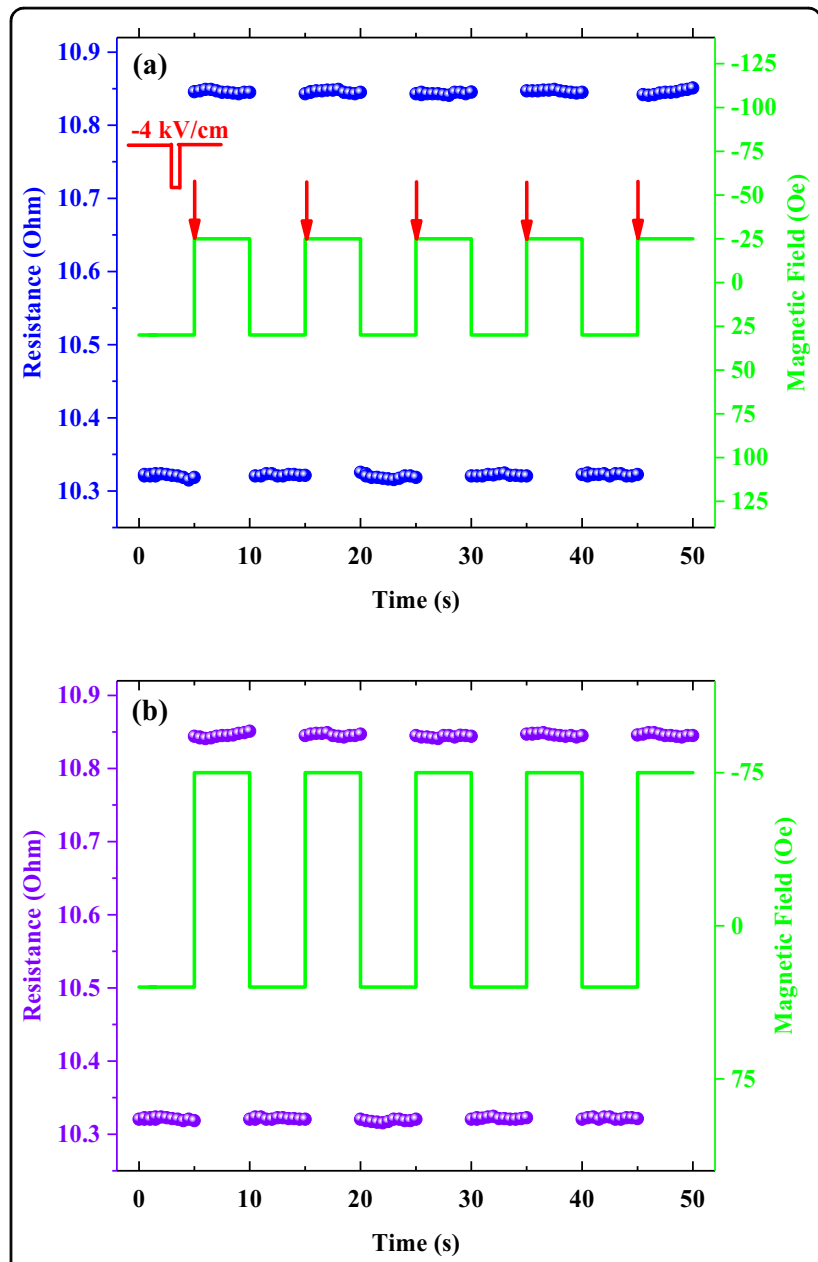

Fig. 6 Dynamic GMR modulation. a Voltage-impulse-assisted GMR modulation under magnetic fields of $-25 \mathrm{Oe}$ and +30 Oe. $\mathbf{b}$ Dynamic GMR modulation under magnetic fields of -75 Oe and +30 Oe.

exerted on the PMN-PT substrate and immediately released (the pulse duration is $10 \mu \mathrm{s}$ ) when the magnetic field is transformed to a negative field at -15 Oe. Surprisingly, the MR rapidly switches from the minimum to maximum value, as shown in Fig. 5 (the blue curve). The magnetic field is reduced by $50 \mathrm{Oe}$ (the green arrow shown in Fig. 5) compared to the initial state.

Here, the voltage impulse induces a volatile strain that rotates the magnetic moment from the positive magnetic field direction toward the [1-10] direction. As the external magnetic field becomes negative, it combines with the strain effective field $H_{\text {eff }}$ to overcome the switching barrier. Normally, the magnitude of the critical negative magnetic field $\left(H_{\text {critical }}\right)$ is decided by the combined magnetic field. As long as the combined magnetic field produced by $H_{\text {eff }}$ and $H_{\text {critical }}$ can overcome the barrier in the $90^{\circ}$ direction, switching can be performed. When the pulse of $-4 \mathrm{kV} / \mathrm{cm}$ is reduced to zero, the strain reverts to zero, and the magnetic 
moments of the free layer orient in the negative direction of the easy axis. Consequently, an antiparallel configuration is obtained. During the switching process, once the magnetic moments overcome the barrier, they do not revert back, and accordingly, a negative magnetic field is also not required to sustain the switching. Therefore, a low-power pulse is extremely helpful in decreasing the switching field and accomplishes energyefficient storage. Figure $5 \mathrm{~b}$ displays the repeated experiments in measuring MR curves at voltageimpulse-assisted states. This demonstrated that this strategy to achieve $180^{\circ}$ magnetization reversal is stable and repeatable.

A completely dynamic GMR modulation upon applying the proper voltage impulse is displayed in Fig. 6a. For the sample, by only adopting the magnetic field to switch, a large field of $-75 \mathrm{Oe}$ is required, as shown in Fig. 6b. However, in voltage-impulse-assisted magnetization reversal, a combined $-4 \mathrm{kV} / \mathrm{cm}$ voltage impulse is applied when a parallel to an antiparallel configuration is required. An ultralow -25 Oe magnetic field is sufficient to achieve these reversals. The switching field is reduced by approximately $70 \%$. Voltage-impulse-assisted magnetization reversal can tremendously reduce the reversal magnetic field. These results represent a crucial step toward ultralow-powerconsumption MRAM devices.

\section{Conclusions}

In summary, we have achieved large (2.5\%), reversible, and nonvolatile E-field-induced MR modulations without any magnetic field in an SV/PMN-PT heterostructure via a strain-mediated ME effect. More significantly, E-fieldassisted $180^{\circ}$ magnetization reversal of the free layer is demonstrated. The threshold magnetic field was reduced by approximately $70 \%$, and the largest MR of $5.5 \%$ was achieved by employing a $10 \mu$ s small voltage impulse. These results provide definite proof that energy-efficient MR memory devices and STT-MRAM with a low writecurrent density can be realized by using an E-field.

\section{Acknowledgements}

This work was supported by the National Natural Science Foundation of China under Grant No. 62071107 and 51772047 and the Science and Technology Department of Sichuan Province under Grant No. 2021YJ0008.

\section{Author contributions}

M.L.L. and X.L.T. conceived and designed the experiments. W.D., H.S., and H.W.Z. conducted the strain-E experiment of the specific PMN-PT substrate and analyzed the theory of producing a nonvolatile strain. W.D., B.L., and H.M. optimized the SV structure and fabrication conditions. M.L.L., H.S., and X.L.T. performed the magnetization rotation experiments and discussed the experimental results. M.L.L. and X.L.T. wrote the manuscript after discussion with all the authors. X.L.T. supervised the entire project.

\section{Data availability}

The data that support the findings of this study are available within the article.

\section{Competing interests}

The authors declare no competing interests.

\section{Publisher's note}

Springer Nature remains neutral with regard to jurisdictional claims in published maps and institutional affiliations.

Received: 23 March 2021 Revised: 21 August 2021 Accepted: 3 September 2021.

Published online: 1 October 2021

\section{References}

1. Wolf, S. A. et al. Spintronics: a spin-based electronics vision for the future. Science 294, 1488-1495 (2001).

2. Ikegawa, S., Mancoff, F. B., Janesky, J. \& Aggarwal, S. Magnetoresistive random access memory: present and future. IEEE Trans. Electron Devices 67, 1407 (2020).

3. Bhatti, S. et al. Spintronics based random access memory: a review. Mater. Today 20, 530-548 (2017).

4. Akerman, J. Toward a universal memory. Science 308, 508-510 (2005).

5. Jorge, P., Junyeon, K., Kouta, K. \& Yoshichika, O. Spintronic devices for energyefficient data storage and energy harvesting. Commun. Mater. 1, 24 (2020).

6. Parkin, S. et al. Magnetically engineered spintronic sensors and memory. Proc. IEEE 91, 661 (2003).

7. Tehrani, S. et al. Progress and outlook for MRAM technology. IEEE Trans. Magn. 35, 2814-2819 (1999).

8. Ramaswamy, R., Lee, J. M., Cai, K. M. \& Yang, H. Recent advances in spin-orbit torques: moving towards device applications. Appl. Phys. Rev. 5, 031107 (2018).

9. Liu, L. Q. et al. Spin-torque switching with the giant Spin Hall effect of tantalum. Science 336, 555-558 (2012).

10. Fukami, S., Anekawa, T., Zhang, C. \& Ohno, H. A spin-orbit torque switching scheme with collinear magnetic easy axis and current configuration. Nat. Nanotechnol. 11, 621 (2010).

11. Cao, Q. et al. Nonvolatile multistates memories for high-density data storage. ACS Appl. Mater. Inter. 12, 42449-42471 (2020).

12. Cai, K. M. et al. Electric field control of deterministic current-induced magnetization switching in a hybrid ferromagnetic/ferroelectric structure. Nat. Mater. 16, 712-716 (2017).

13. Chen, A. T. \& Zhao, Y. G. Progress of converse magnetoelectric coupling effect in multiferroic heterostructures. Acta Phys. Sin. 67, 157513 (2018).

14. Hu, J. M., Chen, L. Q. \& Nan, C. W. Multiferroic heterostructures integrating ferroelectric and magnetic materials. Adv. Mater. 28, 15-39 (2016).

15. Tang, X. L., Su, H., Zhang, H. W. \& Sun, N. X. Voltage-impulse-induced dualrange nonvolatile magnetization modulation in metglas/PZT heterostructure. Appl. Phys. Lett. 109, 202903 (2016).

16. Liu, M. et al. Electrically controlled non-volatile switching of magnetism in multiferroic heterostructures via engineered ferroelastic domain states. NPG Asia Mater. 8, e316 (2016).

17. Childress, J. R. et al. IrMn spin-valves for high density recording. IEEE Trans. Magn. 37, 1745 (2001).

18. Tang, X. L., Zhang, H. W., Su, H., Zhong, Z. Y. \& Jing, Y. L. Changing and reversing the exchange bias in a current-in-plane spin valve by means of an electric current. Appl. Phys. Lett. 91, 122504 (2007).

19. Tehrani, S. et al. Magnetoresistive random access memory using magnetic tunnel junctions. Proc. IEEE 91, 703-714 (2003).

20. Dieny, B. et al. Magnetotransport properties of magnetically soft spin-valve structures. J. Appl. Phys. 69, 4774-4779 (1991).

21. Moodera, J. S. et al. Large magnetoresistance at room temperature in ferromagnetic thin film tunnel junctions. Phys. Rev. Lett. 74, 3273-3276 (1995).

22. Parkin, S. et al. Giant tunnelling magnetoresistance at room temperature with MgO (100) tunnel barriers. Nat. Mater. 3, 862-867 (2004).

23. Chen, A. et al. Giant nonvolatile manipulation of magnetoresistance in magnetic tunnel junctions by electric fields via magnetoelectric coupling. Nat. Commun. 10, 243 (2019)

24. Liu, M. et al. Electric field modulation of magnetoresistance in multiferroic heterostructures for ultralow power electronics. Appl. Phys. Lett. 98, 222509 (2011).

25. Rizwan, S., Zhang, S., Yu, T., Zhao, Y. G. \& Han, X. F. Piezoelectric enhancement of giant magnetoresistance in spin-valves with different magnetic anisotropies. J. Appl. Phys. 113, 023911 (2013). 
26. Huong, D. T., Giang, N., Thuc, V. N. \& Duc, N. H. Electric field-induced magnetoresistance in spin-valve/piezoelectric multiferroic laminates for low-power spintronics. J. Magn. Magn. Mater. 324, 2019-2023 (2012).

27. Wang, L. Q. et al. Electric field-tunable giant magnetoresistance (GMR) sensor with enhanced linear range. ACS Appl. Mater. Inter. 12, 8855-8861 (2020).

28. Chen, A. et al. Angular dependence of exchange bias and magnetization reversal controlled by electric-field-induced competing anisotropies. Adv. Mater. 28, 363-369 (2016).

29. Chen, A. et al. Full voltage manipulation of the resistance of a magnetic tunnel junction. Sci. Adv. 5, eaay5141 (2019).

30. Du, W. et al. Wide range voltage-impulse-controlled nonvolatile magnetic memory in magnetoelectric heterostructure. Appl. Phys. Lett. 117, 222401 (2020).

31. Leal, J. L. \& Kryder, M. H. Spin valves exchange biased by Co/Ru/Co synthetic antiferromagnets. J. Appl. Phys. 83, 3720-3723 (1998).

32. Liu, M. L. et al. Voltage-controlled three-state magnetic memory based on anisotropic magnetoresistance in a multiferroic heterostructure. Appl. Phys. Lett. 116, 152401 (2020).
33. Liu, M. et al. Electrical tuning of magnetism in $\mathrm{Fe}_{3} \mathrm{O}_{4} / \mathrm{PZN}-\mathrm{PT}$ multiferroic heterostructures derived by reactive magnetron sputtering. J. Appl. Phys. 107 073916 (2010).

34. Thiele, C., Dorr, K., Bilani, O., Rodel, J. \& Schultz, L. Influence of strain on the magnetization and magnetoelectric effect in $\mathrm{La}_{0.7} \mathrm{~A}_{0.3} \mathrm{MnO}_{3} / \mathrm{PMN}-\mathrm{PT}$ (001) (A=Sr,Ca). Phys. Rev. B. 75, 054408 (2007).

35. Baek, S. H. et al. Ferroelastic switching for nanoscale non-volatile magnetoelectric devices. Nat. Mater. 9, 309 (2010).

36. Yang, C., Sun, E. W., Yang, B. \& Cao, W. W. Theoretical study on local domain pinning effect due to defect dipole alignment. J. Phys. D 51, 415303 (2018).

37. Du, G. et al. Large stable strain memory effect in poled Mn-doped $\mathrm{Pb}\left(\mathrm{Mn}_{1 / 3} \mathrm{Sb}_{2 / 3}\right) \mathrm{O}_{3}-\mathrm{Pb}(\mathrm{Zr}, \mathrm{Ti}) \mathrm{O}_{3}$ ceramics. Appl. Phys. Lett. 102, 162907 (2013).

38. Su, H. et al. Voltage-impulse-induced three-state non-volatile magnetization switching in FeSiBC/PZT multiferroic heterostructure. J. Alloy. Compd. 685, 546 (2016). 